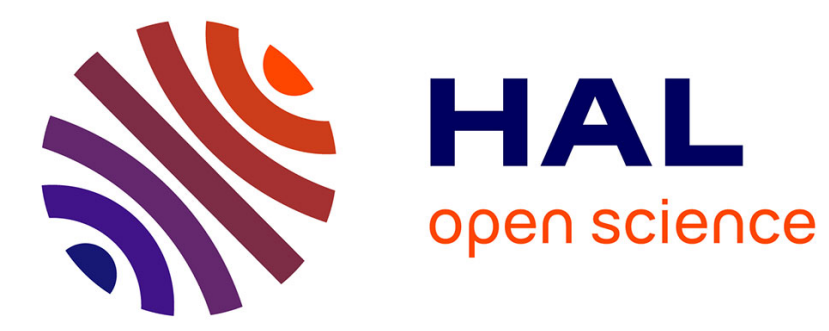

\title{
What happens when cognitive terminals compete for a relaying node?
}

\author{
Elena Veronica Belmega, Brice Djeumou, Samson Lasaulce
}

\section{To cite this version:}

Elena Veronica Belmega, Brice Djeumou, Samson Lasaulce. What happens when cognitive terminals compete for a relaying node?. ICASSP 2009 - 34th IEEE International Conference on Acoustics, Speech and Signal Processing, Apr 2009, Taipei, Taiwan. pp. 2609-2612, 10.1109/icassp.2009.4960157 . hal-00403364

\section{HAL Id: hal-00403364 https://hal.science/hal-00403364}

Submitted on 10 Jul 2009

HAL is a multi-disciplinary open access archive for the deposit and dissemination of scientific research documents, whether they are published or not. The documents may come from teaching and research institutions in France or abroad, or from public or private research centers.
L'archive ouverte pluridisciplinaire HAL, est destinée au dépôt et à la diffusion de documents scientifiques de niveau recherche, publiés ou non, émanant des établissements d'enseignement et de recherche français ou étrangers, des laboratoires publics ou privés. 


\title{
WHAT HAPPENS WHEN COGNITIVE TERMINALS COMPETE FOR A RELAYING NODE?
}

\author{
Elena-Veronica Belmega, Brice Djeumou and Samson Lasaulce \\ LSS (CNRS - SUPELEC - Paris 11) \\ 91190 Gif-sur-Yvette, France \\ \{belmega, djeumou, lasaulce\}@1ss.supelec.fr
}

\begin{abstract}
We introduce a new channel, which consists of an interference channel (IC) in parallel with an interference relay channel (IRC), to analyze the interaction between two selfish and cognitive transmitters who compete for a relay implementing the amplify-and-forward protocol. It is shown that whatever the relay location there is always an equilibrium in the resource allocation game where the users selfishly share their power between the IC and IRC. The uniqueness and determination of this equilibrium is analyzed for two cases: the relay amplification gain is fixed; the IRC direct links are negligible. We show how to exploit this analysis to optimally locate the relay either in terms of individual rate or system sum-rate. Simulations are provided and show, in particular, how the users' selfish behavior leads to sharing the space in regions where the relay is used by only one user or not used at all.
\end{abstract}

Index Terms - Cognitive radio, interference channel, open spectrum access, power allocation game, relay channel.

\section{INTRODUCTION}

Over the last two decades, spectrum congestion has become more and more a critical issue. This is one of the reasons why major actors in this arena like the Federal Communications Commission has released important reports providing a legal framework for deploying technologies like ultra wideband (UWB) or cognitive radio [1]. The latter technology has benefited from a more general consensus in part because its way of re-using the spectrum generates much less interference than UWB systems. Cognitive terminals (CTs), based on spectrum sensing capabilities, are envisioned to be able to opportunistically and efficiently re-exploit the spectrum "left-overs" of other systems. In particular, more and more wireless devices operate in unlicensed bands, which gives to CTs a particular interest in locally exploiting the unused spectral resources, for instance, to boost they transmission rate or quality of communication. The technical issues addressed in this paper fall within this framework. More specifically, our main motivation is to acquire a better understanding of a system where two cognitive transmitters, each of them communicating with their respective receivers, are offered the opportunity to use a relay operating in its own frequency band. We assume that these two transmitters can choose freely their resource allocation policies in order to selfishly maximize their transmission rates and, for this purpose, compete for the available relay node. Several key questions arise: Is there a predictable state (equilibrium) at which this system will operate? Is it unique? How is interference self-managed by selfish and free users? For a given propagation scenario, what is the point at which the relay should be located to optimize a given performance metric?

The system under investigation can be modeled by a frequency non-selective (FNS) interference channel (IC) [2] in parallel with an FNS interference relay channel (IRC) [3, 4]. To the best authors' knowledge, the closest contributions to those presented in this paper are $[3,4,5,6]$. In [5] the authors consider the multiuser power control problem in a frequency-selective IC, which is modeled as a noncooperative game where the players want to maximize their individual transmission rates. In [6] the authors study the same channel but in their formulation the users selfishly minimize their transmit power under a minimal achievable rate constraint. In [3, 4] the authors introduce the IRC and focus on evaluating achievable rate regions for this channel when the links are assumed to be FNS. Here, we not only want to study a new channel but we also adopt a different point of view than [3,4]. Indeed the game-theoretic formulation of the problem is particularly relevant here since the users are not only interacting because of interference in each of the two sub-channels, but they are also cognitive and therefore able to observe their environment and react to it accordingly.

This paper is structured as follows. We introduce the general system model in Sec. 2. We define and analyze the corresponding power allocation (PA) game in Sec. 3. Then we provide a thorough analysis of two cases of practical interest: the case where the relay amplification gain is fixed (Sec. 4); the case where the IRC direct links are negligible (Sec. 5). In Sec. 6, we introduce a Stackelberg formulation to optimize the relay location. Our analysis is illustrated by simulations in Sec. 7. Conclusions are given in Sec. 8 .

\section{GENERAL SYSTEM MODEL}

The system under investigation comprises two source nodes $\mathcal{S}_{1}, \mathcal{S}_{2}$ sending their message to their respective destination node $\mathcal{D}_{1}, \mathcal{D}_{2}$ in a certain frequency band (denoted by (a)), which is assumed to be unitary. Additionally there is one relaying node $\mathcal{R}$ that is available on an additional and orthogonal frequency band (denoted by (b)), also unitary. The signals transmitted by $\mathcal{S}_{1}$ and $\mathcal{S}_{2}$ in the bands (a) and (b), denoted by $X_{1}^{(a)}, X_{1}^{(b)}, X_{2}^{(a)}, X_{2}^{(b)}$, are assumed to be independent and subject to power constraints: $\forall i \in\{1,2\}, \mathbb{E}\left|X_{i}^{(a)}\right|^{2}+$ $\mathbb{E}\left|X_{i}^{(b)}\right|^{2} \leq P_{i}$. The relay implements a zero-delay scalar amplifyand-forward (AF) protocol and operates in the full-duplex mode (we propose a way of making this assumption reasonable in Sec. 5) and subject to the constraint $\mathbb{E}\left|X_{r}^{(b)}\right|^{2} \leq P_{r}$. Under these assumptions, and denoting by $g_{i j}, h_{i j}$ the channel gains between $\mathcal{S}_{i}, \mathcal{D}_{j}$ and $\mathcal{R}$ on bands (a) and (b) respectively, the received baseband signals write:

The authors would like to thank the RAF project (SYSTEMATIC), sponsored by Région Ile-de-France and the French Ministry of Industry. 


$$
\left\{\begin{array}{l}
Y_{1}^{(a)}=g_{11} X_{1}^{(a)}+g_{21} X_{2}^{(a)}+Z_{1}^{(a)} \\
Y_{2}^{(a)}=g_{22} X_{2}^{(a)}+g_{12} X_{1}^{(a)}+Z_{2}^{(a)} \\
Y_{r}^{(b)}=h_{1 r} X_{1}^{(b)}+h_{2 r} X_{2}^{(b)}+Z_{r}^{(b)} \\
Y_{1}^{(b)}=a_{r} h_{r 1} Y_{r}^{(b)}+h_{11} X_{1}^{(b)}+h_{21} X_{2}^{(b)}+Z_{1}^{(b)} \\
Y_{2}^{(b)}=a_{r} h_{r 2} Y_{r}^{(b)}+h_{22} X_{2}^{(b)}+h_{12} X_{1}^{(b)}+Z_{2}^{(b)}
\end{array}\right.
$$

We consider a realistic situation where only large scale propagation effects can be taken into account by the users to optimize their rate. Thus the channel gains are considered non time-varying. Concerning channel state information (CSI), we will always assume coherent communications between each pair $\left(\mathcal{S}_{i}, \mathcal{D}_{i}\right)$ whereas at the transmitters the information assumptions will be context-depending and therefore provided in the corresponding sections. The variances of the zero-mean additive white Gaussian complex noises $Z_{k}^{(a)}$, $Z_{k}^{(b)}, Z_{r}^{(b)}$ with $k \in\{1,2\}$ are denoted by $N_{k}^{(a)}, N_{k}^{(b)}, N_{r}^{(b)}$. At last $a_{r}$ corresponds to the relay amplification gain and is, in general, a function of $\theta_{1}$ and $\theta_{2}$ where $\forall i \in\{1,2\}, \theta_{i} P_{i} \triangleq \mathbb{E}\left|X_{i}^{(b)}\right|^{2}$. As the relay is subject to a power constraint the amplification gain $a_{r}\left(\theta_{1}, \theta_{2}\right)$ cannot be chosen arbitrarily. In Sec. 3 and 5 we choose $a_{r}=\sqrt{\frac{P_{r}}{\mathbb{E}\left|Y_{r}^{(b)}\right|^{2}}}=\sqrt{\frac{P_{r}}{\left|h_{1 r}\right|^{2} P_{1} \theta_{1}+\left|h_{2 r}\right|^{2} P_{2} \theta_{2}+N_{r}^{(b)}}}$. In this way one is ensured that the relay makes use of all its available power while respecting the limiting constraint. However, in Sec. 4 we choose $a_{r}=$ const. $=a_{r}(1,1)$. To conclude this section note that will call the state of the network the vector of power fractions that the users allocate to the IRC i.e. $\underline{\theta}=\left(\theta_{1}, \theta_{2}\right)$.

\section{GENERAL POWER ALLOCATION GAME}

One of our goals is to know how the transmitters are going to allocate their powers between the the IC and IRC, given the fact that are able to observe each other and react accordingly. This situation of interaction corresponds to a game, and more precisely to a noncooperative strategic-form game. The players of the game are the two transmitters. The strategy of transmitter $i$ consists in choosing $\theta_{i}$ in his strategy set $\mathcal{A}_{i}=[0,1]$ in order to maximize his individual rate, which is chosen to be his utility. Note that we implicitly assume Gaussian codebooks for the two users since this choice is optimum for both or them. The utility function for user $i \in\{1,2\}$ is thus given by $u_{i}=R_{i}^{(a)}+R_{i}^{(b)}$ where $R_{i}^{(a)}=C\left(\eta_{i}^{(a)}\right), R_{i}^{(b)}=C\left(\eta_{i}^{(b)}\right)$, $C(x)=\log _{2}(1+x)$ is the capacity function and

$$
\left\{\begin{aligned}
\eta_{i}^{(a)} & =\frac{\left|g_{i i}\right|^{2} \rho_{i}^{(a)} \overline{\theta_{i}}}{\left|g_{j i}\right|^{2} \rho_{j}^{(a)} \frac{N_{j}^{(a)}}{N_{i}^{(a)}} \overline{\theta_{j}}+1} \\
\eta_{i}^{(b)} & =\frac{\left|a_{r} h_{i r} h_{r i}+h_{i i}\right|^{2} \rho_{i}^{(b)} \theta_{i}}{\left|a_{r} h_{j r} h_{r i}+h_{j i}\right|^{2} \rho_{j}^{(b)} \frac{N_{j}^{(b)}}{N_{i}^{(b)}} \theta_{j}+a_{r}^{2}\left|h_{r i}\right|^{2} \frac{N_{r}^{(b)}}{N_{i}^{(b)}}+1}
\end{aligned}\right.
$$

with $\forall(i, s) \in\{1,2\} \times\{a, b\}, \rho_{i}^{(s)}=\frac{P_{i}}{N_{i}^{(s)}}$ and $j=-i$ (standard notation to refer to the whole set of players except for player $i$ ). In order to gain in terms of clarity, WLOG we will assume in the sequel that $\forall(i, s) \in\{1,2\} \times\{a, b\}, N_{i}^{(s)}=N_{r}^{(b)}=N$ and therefore introduce the quantities $\forall i \in\{1,2, r\}, \rho_{i}=\frac{P_{i}}{N}$.

As mentioned in the introduction a desirable feature of networks where users take their decisions by themselves is the existence of one or several states at which users will operate with a certain degree of stability. In this respect, the Nash equilibrium (NE) [7] corresponds to a state of the network that verifies the minimum condition of stability i.e. if one user deviates from this state while the other users do not deviate from it, he looses in terms of utility, and therefore returns to his original strategy. This translates mathematically by the following definition.

Definition 3.1 (Nash equilibrium) The state $\underline{\theta}^{*}$ is a pure $N E$ if $\forall i \in\{1,2\}, \forall \theta_{i}^{\prime} \in \mathcal{A}_{i}, u_{i}\left(\theta_{i}^{*}, \theta_{-i}^{*}\right) \geq u_{i}\left(\theta_{i}^{\prime}, \theta_{-i}^{*}\right)$.

Is there such an equilibrium in the proposed system? The answer is given through the following theorem.

Theorem 3.2 (Existence of an NE) Let $\widehat{u}_{i}=R_{i}^{(a)}+\widehat{R}_{i}^{(b)}$ be the modified utility function for user $i$, where $\widehat{R}_{i}^{(b)}$ is the transmission rate user $i$ obtains on band $(b)$ by transmitting on it with power $\frac{\theta_{i} P_{i}}{\alpha_{i}}$ during a fraction of time $\alpha_{i} \in[0,1]$ and with zero power for the rest of the time. Assuming these utility functions, there will always be an $N E$ in the general PA game.

The proof of this theorem is based on Theorem 1 of [8]. In [8] Rosen provides sufficient conditions for the existence of an NE. If, for every player 1) his strategy set is convex and compact, 2) his utility is continuous in the vector of strategies and 3) concave in his own strategy, then the existence of at least one NE is guaranteed. In our setup it is easy to check that conditions 1) and 2) are met both for $\widehat{u}_{i}$ and $u_{i}$. However checking condition 3 ) for the utility $u_{i}$ leads to expressions that are not easy to manipulate. This is the reason why we introduced the new utility $\widehat{u}_{i}$. Indeed, as we want the user utility to be his (Shannon) transmission rate, it necessarily has to be a concave function of $\theta_{i} P_{i}$. Therefore, even if the achievable rate $u_{i}$ turned out to be non-concave, user $i$ can always apply a time-sharing procedure for the rate $R_{i}^{(b)}$ to make it concave, which is precisely what is done in Theorem 4.1.

The uniqueness issue is however more difficult to be dealt with properly, especially because of space limitations. In particular, proving the diagonal strict concavity condition from Theorem 2 by Rosen [8] is much more mathematically demanding. For this reason and because the general PA game is also the most demanding one in terms of CSI at the transmitters, we will only present simulation results for this case and rather provide a thorough theoretical analysis of two special but practical cases. After observing many simulation results and analyzing several special cases of the general game, we conjecture that the uniqueness is not guaranteed only in very special scenarios that have little chance to occur.

\section{CASE OF A FIXED AMPLIFICATION GAIN}

Although choosing $a_{r}\left(\theta_{1}, \theta_{2}\right)$ as above allows the relay to exploit all its power, it involves some knowledge on the channels at $\mathcal{S}_{1}, \mathcal{S}_{2}, \mathcal{R}$ and a certain relay structure, which is not always available. In particular, it assumes at the relay the presence of a mechanism to estimate the power of the received signal. While this can be easy for a digital relay transceiver that knows the possible training sequences used by the sources, it might be impossible if the relay is imposed to be a (dummy) analog power amplifier. At the sources, the knowledge in terms of channel gains (path losses) depends on the way the PA algorithm is implemented. In the case where the Cournot tâtonnement process (see e.g. [9]) converges, user $i$ needs to know $\forall(k, s) \in\{1,2\} \times\{a, b\}, \rho_{k}^{(s)}, P_{r}, g_{i i}, h_{i i}, h_{i r}, h_{r i}$ and $h_{j j}$ and $h_{j r}$ with $j=-i$. This process will be detailed further. What matters at this point is that, depending on the amplification gain chosen, the degree of knowledge at the terminals can be more a less severe. In 
this section we assume that $a_{r}$ is a certain constant w.r.t. $\underline{\theta}$, denoted by $A_{r}$, and meets the power constraint. An interesting issue, a possible extension, is the analysis of the optimal amplification gain and whether saturating the relay power constraint may be suboptimal. Here, we restrict our attention on the case where $A_{r}=a_{r}(1,1)$ which is a simple choice meeting the relay power constraint and can be improved if some statistical information is available. As the case $a_{r}=A_{r}$ is a special case of the general PA game analyzed in Sec. 3 the existence of an NE is guaranteed thanks to Theorem 3.2. In fact, in the special case under investigation the concavification procedure is not necessary and $u_{i}$ can be shown to be a concave function of $\theta_{i}$. As for the uniqueness issue, assuming no constraints on the users' strategies, the following theorem provides the condition under which it is ensured and corresponding selfish PA policy.

Theorem 4.1 (Uniqueness of the NE when $a_{r}(\underline{\theta})=A_{r}$ ) For $(i, j) \in\{(1,2),(2,1)\}$, let $\alpha_{i i}=2\left|g_{i i}\right|^{2}\left|A_{r} h_{r i} h_{i r}+h_{i i}\right|^{2} \rho_{i}$, $\alpha_{i j}=\left|g_{j i}\right|^{2}\left|A_{r} h_{r i} h_{i r}+h_{i i}\right|^{2} \rho_{j}+\left|g_{i i}\right|^{2}\left|A_{r} h_{r i} h_{j r}+h_{j i}\right|^{2} \rho_{j}$ and $\beta_{i}=\left|A_{r} h_{r i} h_{i r}+h_{i i}\right|^{2}\left(1+\left|g_{i i}\right|^{2} \rho_{i}+\left|g_{j i}\right|^{2} \rho_{j}\right)-\left|g_{i i}\right|^{2}(1+$ $\left.A_{r}^{2}\left|h_{r i}\right|^{2}\right)$. The game has a unique NE if and only if $\alpha_{11} \alpha_{22}-$ $\alpha_{12} \alpha_{21} \neq 0$, given by: $\theta_{1}^{*}=\frac{\alpha_{22} \beta_{1}-\alpha_{12} \beta_{2}}{\alpha_{11} \alpha_{22}-\alpha_{12} \alpha_{21}}$ and $\theta_{2}^{*}=\frac{\alpha_{11} \beta_{2}-\alpha_{21} \beta_{1}}{\alpha_{11} \alpha_{22}-\alpha_{12} \alpha_{21}}$

Note that the assumed condition is mild since it would not be met in very special configurations of the network (e.g. a symmetric network). To prove the claimed result we exploit the notion of best responses (BR). The BR of player $i$ to player $j$ is defined by $\mathrm{BR}_{i}\left(\theta_{j}\right)=\arg \max _{\theta_{i}} u_{i}(\underline{\theta})$. In general it is a correspondence but in our case it is just a function. The equilibrium points precisely correspond to the intersection of the BRs of the two users. It turns out that, for each $i \in\{1,2\}$, the $\mathrm{BR}$ is an affine function of $\theta_{j}$ with $j=-i$, which means that we are in the same situation as in a Cournot duopoly [10]. It can be checked that $\operatorname{BR}_{i}\left(\theta_{j}\right)=-\frac{\alpha_{i j}}{\alpha_{i i}} \theta_{j}+\frac{\beta_{i}}{\alpha_{i i}}$ with $j=-i$. The provided optimum selfish PA for each user follows. Now, in order to take into consideration that $\theta_{i} \in[0,1]$, one just needs to introduce four Lagrangian multipliers and integrate the KKT conditions to impose the associated constraints.

So far, we have treated the issues of existence, uniqueness and determination of the NE. By inspecting the solution given by Theorem 4.1, we see that each user needs to know all channel gains of the system to optimally exploit the spectrum. Although these gains correspond to path losses and could be easily acquired in scenarios where they do not vary rapidly over time, it is desirable to relax the degree of knowledge at the sources. This is precisely what the Cournot tâtonnement process allows one to do. The idea is that one player observes the strategy played by the other player and reacts accordingly by maximizing his BR and so on. In our context, as the BRs are affine functions, one is ensured that from any starting state $\left(\theta_{1}^{0}, \theta_{2}^{0}\right)$ the procedure will converge to the NE. What does a source node need to know to implement such a procedure? User $i$ needs to know the path losses $g_{i i}, h_{i i}, h_{i r}, h_{r i}, h_{j r}$, all the signal-to-noise ratios $\rho_{i}$, the average level of interference plus noise he undergoes on each band and of course the strategy played by the other player $\theta_{j}$. The latter quantity can be acquired by a sensing technique based on a standard estimation procedure, which is particularly easy in fast fading environment since the path loss can be considered to be constant during a period of time long enough to store a large number of channel realizations. The other quantities can be acquired through feedback or sensing mechanisms.

\section{CASE OF NEGLIGIBLE DIRECT LINKS}

We start this section by providing some motivations for studying the case where, in the IRC, the signals received through the direct links can be neglected w.r.t. the signal received from the relay. In some contexts, especially those where the relay node is fully exploited, the contribution of the direct link to the received signal can be marginal. This can happen in environments where the path loss exponents associated with band (b) are relatively high (typically $>3$ ). Another situation of practical interest is the case where the relay is composed of a receiver and transmitter that are not co-located (e.g. if they are located at the opposite edges of a building) and the corresponding antennas implement orthogonal polarizations (say in order to reasonably meet the full-duplex requirement). In the latter case, the destination would use a polarization orthogonal to that used by the source, making its contribution totally negligible in comparison to that of the relay. As the amplification gain assumed here is the one used in Sec. 3, which saturates the power constraint at the relay. When these propagation conditions are met, the rates of the users can be approximated by: $\tilde{u}_{i}(\underline{\theta})=C\left(\tilde{\eta}_{i}^{(a)}\right)+C\left(\tilde{\eta}_{i}^{(b)}\right)$ with $\tilde{\eta}_{i}^{(a)}=\frac{\left|g_{i i}\right|^{2} \rho_{i} \overline{\theta_{i}}}{\left|g_{j i}\right|^{2} \rho_{j} \bar{\theta}_{j}+1}, \tilde{\eta}_{i}^{(b)}=$ $\frac{\left|h_{1 r} h_{r 1}\right|^{2} \rho_{r} \rho_{1} \theta_{1}}{\left|h_{1 r}\right|^{2} \rho_{1} \theta_{1}+\left(\left|h_{2 r} h_{r 1}\right|^{2} \rho_{r}+\left|h_{2 r}\right|^{2}\right) \rho_{2} \theta_{2}+\left|h_{r 1}\right|^{2} \rho_{r}+1}$, always with $j=$ $-i$. Here again each rate $\tilde{u}_{i}$ is already concave in $\theta_{i}$ and Theorem 3.2 can be applied without time-sharing. In this case the BRs are given by $B R_{i}\left(\theta_{j}\right)=-\frac{\beta_{i}\left(\theta_{j}\right)+\sqrt{\Delta_{i}\left(\theta_{j}\right)}}{2}$, where $\Delta_{i}\left(\theta_{j}\right)=\beta_{i}^{2}\left(\theta_{j}\right)-$ $4 \alpha_{i} \tau_{i}\left(\theta_{j}\right)$, and $\alpha_{i}=\left|g_{i i}\right|^{2}\left|h_{i r}\right|^{4}\left(1+\left|h_{r i}\right|^{2} \rho_{r}\right) \rho_{i}^{2}, \quad \beta_{i}\left(\theta_{j}\right)=$ $2\left|g_{i i}\right|^{2}\left|h_{i r}\right|^{2}\left(1+\left|h_{r i}\right|^{2} \rho_{r}\right) \rho_{i} f_{i}\left(\theta_{j}\right), \tau_{i}\left(\theta_{j}\right)=\left|g_{i i}\right|^{2} f_{i}^{2}\left(\theta_{j}\right)-$ $\left|h_{r i}\right|^{2}\left|h_{i r}\right|^{2}\left[\left|g_{i i}\right|^{2} \rho_{i}+1+\left|g_{j i}\right|^{2}\left(1-\theta_{j}\right) \rho_{j}\right] \rho_{r} f_{i}\left(\theta_{j}\right), f_{i}\left(\theta_{j}\right)=$ $\left(\left|h_{j r} h_{r i}\right|^{2} \rho_{r}+\left|h_{j r}\right|^{2}\right) \rho_{j} \theta_{j}+\left|h_{r i}\right|^{2} \rho_{r}+1$. It turns out that it is possible to analyze the properties of these BRs to show that the uniqueness of the NE is generically guaranteed.

\section{OPTIMIZING THE RELAY LOCATION}

As the relay location is a determinant factor for the performance of the users and overall system, we propose here a Stackelberg formulation of the problem. We introduce a leader in the game whose strategy is the pair $\left(x_{\mathcal{R}}, y_{\mathcal{R}}\right)$, which indicates the relay location when assuming a $2 \mathrm{D}$ propagation scenario. We consider two choices for the leader's utility: A) the system sum-rate $\left.u\left(x_{\mathcal{R}}, y_{\mathcal{R}}\right)=u_{1}\left[\underline{\theta}^{*}\left(x_{\mathcal{R}}, y_{\mathcal{R}}\right)\right]+u_{2}\left[\underline{\theta}^{*}\left(x_{\mathcal{R}}, y_{\mathcal{R}}\right)\right], \mathrm{B}\right)$ the rate of a given user (1 or 2). Choice A) would correspond, for example, to the case where the system is owned by the same provider or where an agreement between two providers would have been found whereas B) would correspond to the case where one of the users would be able to choose the relay location (picture one WiFi subscriber who wants to increase his downlink throughput by placing his cellular phone somewhere in his apartment while his neighbor can also exploit the same spectral resources). Then, the sources (the followers of the game) react to the leader's strategy by choosing their best selfish PA policies. Interestingly, we know from Theorem 3.2 that for any location of the relay there will be an equilibrium. The goal of the leader is to make this equilibrium efficient in the sense of A) or $\mathrm{B})$.

\section{SIMULATION RESULTS}

We consider a square cell with side length $2 L, L=10 \mathrm{~m}$. The source and destination nodes are fixed and located in $[-L, L]^{2}$ as follows: $\mathcal{S}_{1}:\left(-\frac{3 L}{8}, \frac{L}{4}\right), \mathcal{S}_{2}:\left(-\frac{L}{4},-\frac{3 L}{8}\right), \mathcal{D}_{1}:\left(\frac{L}{4}, \frac{L}{16}\right), \mathcal{D}_{2}:$ 


\begin{tabular}{|l|c|c|c|c|c|c|c|c|}
\hline$\gamma^{(a)}$ & 2 & 2 & 2 & 3 & 3 & 3 & 4 & 4 \\
\hline$\gamma^{(b)}$ & 2 & 3 & 4 & 2 & 3 & 4 & 2 & 4 \\
\hline$P_{r}=30$ & 3.15 & 1.51 & 1 & 3.42 & 4.96 & 1.5 & 3.42 & 6.32 \\
\hline$P_{r}=20$ & 1.9 & 1 & 1 & 1.94 & 2 & 1 & 1.94 & 2 \\
\hline
\end{tabular}

Table 1. Performance gains brought by adding a relay on the IRC

$\left(\frac{3 L}{8},-\frac{L}{8}\right)$. Fig. 1 is a temperature image representing the system sum-rate intensity for which a given pixel corresponds to a given location of the relay in the scenario: $P_{1}=20 \mathrm{dBm}, P_{2}=17 \mathrm{dBm}$, $P_{r}=27 \mathrm{dBm}, N_{1}^{(a)}=N_{1}^{(b)}=10 \mathrm{dBm}, N_{2}^{(a)}=N_{2}^{(b)}=9 \mathrm{dBm}$, $N_{r}^{(b)}=7 \mathrm{dBm}$ and the path loss exponents $\gamma^{(a)}=2, \gamma^{(b)}=2.5$. This scenario and all the (numerous) scenarios we analyzed showed that the optimal location for the relay is on one of the two segments originating from two sources to their respective destination. The question of which segment is the best and where to put the relay on this segment can be determined (at least) numerically. Even though space is missing to fully interpret and possibly prove this result, we will just mention that this can be roughly explained by the fact that the individual rate of a given user is maximized on the segment between him and his destination, which results from the optimal (selfish) tradeoff between the strength of the received signal and the amount of interference he undergoes. Fig. 2 is a temperature image representing the power fraction $\theta_{i}$ user $i$ allocates to the IRC for the different positions of the relay in the cell for the scenario: $P_{1}=P_{2}=17 \mathrm{dBm}, P_{r}=23 \mathrm{dBm}, N_{1}^{(a)}=N_{1}^{(b)}=10 \mathrm{dBm}$, $N_{2}^{(a)}=N_{2}^{(b)}=9 \mathrm{dBm}, N_{r}^{(b)}=7 \mathrm{dBm}, \gamma^{(a)}=2, \gamma^{(b)}=3$. Interestingly, this typical scenario shows that the regions where the transmitters uses some power to use the relay do not overlap. This means that, the selfish behavior of the users towards using the relay is self-regulated, which naturally leads to delineating frontiers above which the relay is left for the other user or not used at all. We also provide Tab. 7 which shows the influence of the path loss exponents, the relay power level $(\mathrm{dBm})$ on the ratio of sum-rate with relay to that with no relay for: $N_{1}^{(a)}=7 \mathrm{dBm}, N_{2}^{(a)}=9 \mathrm{dBm}$, $N_{1}^{(b)}=N_{2}^{(b)}=N_{r}=10 \mathrm{dBm}, P_{1}=20 \mathrm{dBm}, P_{2}=22 \mathrm{dBm}$. At last, we would like to mention that other simulations have shown that introducing fading does not change our conclusions provided that ergodic rates are considered.

\section{CONCLUSION}

We have studied a simplified scenario of the general situation where a set of source-destination pairs coexist on the same unlicensed band and can opportunistically exploit an additional band for which relay nodes are available. We have seen that it is not only possible to show the existence of an equilibrium in the proposed scenario, but also that it is generically unique and it is possible to determine it. It is also possible to determine the optimum relay location for a given performance index and analyze the way the interference is self-managed by the users in such a network. This shows that in unlicensed networks with multiple relay nodes, the selfish behavior of cognitive users could self-regulate the amount of interference in the network. The approach introduced in this work should therefore be generalized to more complex networks in terms of topology but also by introducing mobility and different relaying protocols.

\section{REFERENCES}

[1] "Report of the Spectrum Efficiency Working Group", Federal Communications Commision, Nov. 2002.

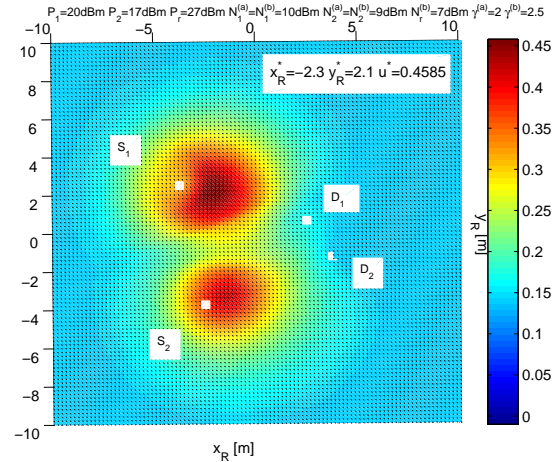

Fig. 1. System sum-rate at the NE for $\left(x_{\mathcal{R}}, y_{\mathcal{R}}\right) \in[-10,10]^{2}$.

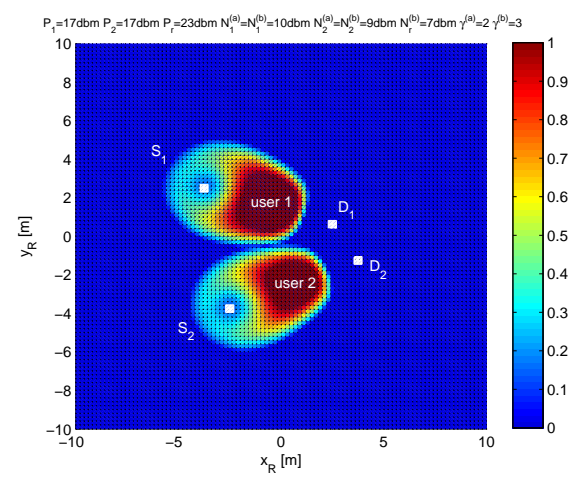

Fig. 2. Selfish PA policies at the NE: $\theta_{i}^{*}$ for $\left(x_{\mathcal{R}}, y_{\mathcal{R}}\right) \in[-10,10]^{2}$.

[2] A. B. Carleial, "Interference Channels", IEEE Trans. on Inform. Theory, Vol. 24, No. 1, pp. 66-70, Jan. 1978.

[3] O. Sahin and E. Erkip, "On Achievable Rates for Interference Relay Channel with Interference cancellation", Asilomar Conf. on Signals, Systems and Computers, pp. 805-809, Nov. 2007.

[4] O. Sahin and E. Erkip, "Achievable Rates for the Gaussian Interference Relay Channel”, Globecom, pp. 1627-1631, 2007.

[5] W. Yu, G. Ginis and J. M. Cioffi, "Distributed multiuser power control for digital subscriber lines", IEEE J. of Sel. Areas in Comm., Vol. 20, No. 5, pp. 1105-1115, June 2002.

[6] J.-S. Pang, G. Scutari, F. Facchinei, C. Wang, "Distributed Power Allocation with Rate Constraints in Gaussian Parallel Interference Channels", IEEE Trans. on Inform. Theory, Vol. 54, No. 8, pp. 3471-3489, Aug. 2008.

[7] J. F. Nash, "Equilibrium points in n-points games", Proc. of the Nat. Academy of Science, Vol. 36, no. 1, pp. 48-49, Jan. 1950.

[8] J. Rosen, "Existence and uniqueness of equilibrium points for concave n-person games", Econometrica, Vol. 33, pp. 520-534, 1965.

[9] H. Moulin, "Dominance Solvability and Cournot Stability", Mathematical Social Sciences, Vol. 7, pp. 83-102, 1984.

[10] A. Cournot, "Recherches sur les principes mathématiques de la la théorie des richesses, 1838, re-edited by Mac Millan in 1987. 